Jennifer Cecilia Ordóñez-León; Darwin Gabriel García-Herrera; Sergio Constantino Ochoa-Encalada; Juan Carlos Erazo-Álvarez

http://dx.doi.org/10.35381/r.k.v5i5.1036

\title{
Educación emocional y su influencia como estrategia educativa con estudiantes de bachillerato
}

\author{
Emotional education and its influence as an educational strategy with high \\ school students
}

Jennifer Cecilia Ordóñez-León

jordonez@ucacue.edu.ec

Universidad Católica de Cuenca, Azogues

Ecuador

https://orcid.org/0000-0002-8123-1569

Darwin Gabriel García-Herrera dggarciah@ucacue.edu.ec

Universidad Católica de Cuenca, Azogues

Ecuador

https://orcid.org/0000-0001-6813-8100

Sergio Constantino Ochoa-Encalada

scochoae@ucacue.edu.ec

Universidad Católica de Cuenca, Cuenca

Ecuador

https://orcid.org/0000-0003-3067-3719

Juan Carlos Erazo-Álvarez

jcerazo@ucacue.edu.ec

Universidad Católica de Cuenca, Cuenca

Ecuador

https://orcid.org/0000-0001-6480-2270

Recibido: 15 de septiembre de 2020

Revisado: 19 de octubre de 2020

Aprobado: 15 de noviembre de 2020

Publicado: 01 de diciembre de 2020 
Revista Arbitrada Interdisciplinaria KOINONIA

Año 2020. Vol V. N5. Especial II: Educación

Hecho el depósito de Ley: FA2016000010

ISSN: 2542-3088

FUNDACIÓN KOINONIA (F.K). Santa Ana de Coro. Venezuela.

Jennifer Cecilia Ordóñez-León; Darwin Gabriel García-Herrera; Sergio Constantino Ochoa-Encalada; Juan Carlos Erazo-Álvarez

\title{
RESUMEN
}

El objetivo de la investigación es analizar la educación emocional y su influencia como estrategia educativa con estudiantes de bachillerato del Ecuador. Metodológicamente fue de tipo descriptiva no experimental, su enfoque fue cuantitativo y de cohorte transversal. La relación de variables sometidas a Chi cuadrado indican una hipótesis afirmativa con un valor de P 0,040 menor a 0,05, entre si los estudiantes piensan que merece la pena prestar atención a sus emociones y estado de ánimo y la importancia de la relación con sus docentes. La propuesta planteada de planificar E.M.M. en el aula como un eje transversal en las asignaturas impartidas en el nivel de estudio es, considerarlo como un componen te esencial que coadyuvará a la mejora académica y conductual de estudiantes, pretendiendo que su aplicación genere replicas satisfactorias en aquellos docentes que aun $\mathrm{n}$ o han tenido el acercamiento hacia dicho conocimiento.

Descriptores: Proceso de aprendizaje; psicología de la educación; rendimiento escolar. (Palabras tomadas del Tesauro UNESCO).

\begin{abstract}
The objective of the research is to analyze emotional education and its influence as an educational strategy with high school students from Ecuador. Methodologically, it was descriptive, non-experimental, its approach was quantitative and cross-sectional cohort. The relationship of variables subjected to Chi square indicates an affirmative hypothesis with a value of $P 0.040$ less than 0.05 , between whether students think that it is worth paying attention to their emotions and mood and the importance of the relationship with their teachers. The proposed proposal to plan E.M.M. in the classroom as a transversal axis in the subjects taught at the study level is to consider it as an essential component that will contribute to the academic and behavioral improvement of students, pretending that its application generates satisfactory replications in those teachers who have not yet had the approach towards such knowledge.
\end{abstract}

Descriptors: Learning processes; educational psychology; academic achievement. (Words taken from the UNESCO Thesaurus). 


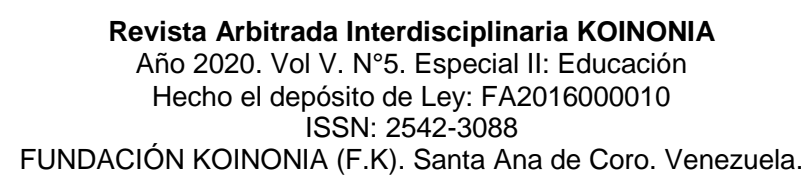

Jennifer Cecilia Ordóñez-León; Darwin Gabriel García-Herrera; Sergio Constantino Ochoa-Encalada; Juan Carlos Erazo-Álvarez

\section{INTRODUCCIÓN}

La educación tradicional habitualmente se ha enfocado en el desarrollo de habilidades cognitivas, centrando su efecto en ello, sin embargo en la actualidad los docentes desafían diferentes situaciones que coartan el éxito del proceso de enseñanza pues, si se considera la diversidad que existe dentro de un aula, los estudiantes presentan dificultades no solo de índole académica y/o conductuales, además, están las emocionales, siendo esta última la menos atendida por el sistema educativo en general. Es evidente que dentro del aula de clase ocurren diversos conflictos que están vinculados directamente hacia el estado emocional de los estudiantes, provocando bajo rendimiento académico o deserciones escolares, problemas conductuales dentro del aula, acarreando falta de respeto entre ellos y hacia el profesor, en muchas ocasiones inasistencia recurrente, estudiantes con escaso control en su disciplina, violencia no verbal y verbal entre pares, discusiones que inicialmente podrían ser controladas, sin embargo no se llega a consensos, baja autoestima, entre otros, obedeciendo a una bajo control de sus emociones o desconocimiento de las mismas.

En referencia a datos presentados por (Organización de las Naciones Unidas para la Educación, la Ciencia y la Cultura [UNESCO], 2020), la deserción de los estudiantes denota un grave problema recurrente en las instituciones educativas a nivel de Latinoamérica, provocando un impacto negativo en el desarrollo normal de la sociedad, si consideramos que uno de los factores que podrían impulsar esta deserción sería la defectuosa relación implícita entre los educandos y educadores, según (ArizaHernández, 2017), quien refiere sus estudios en la in fluencia que tiene la relación que se genere entre docentes y estudiantes, podríamos generar ya un punto de partida, para poder fortalecer este vínculo afectivo, mediante la puesta en práctica de la educación emocional como estrategia educativa en el aula.

La propuesta de (Barrientos-Fernández, et al., 2019), quienes sugieren la implementación de estrategias en el aula, por parte del profesorado, motivando a la adquisición y puesta en práctica de habilidades y destrezas en el control de emociones 


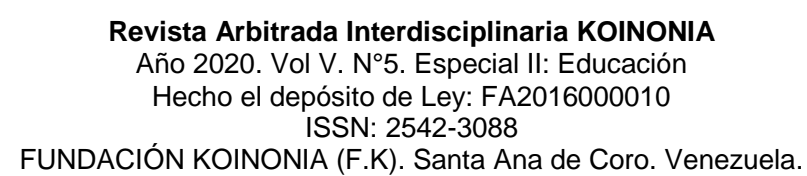

Jennifer Cecilia Ordóñez-León; Darwin Gabriel García-Herrera; Sergio Constantino Ochoa-Encalada; Juan Carlos Erazo-Álvarez

en los estudiantes, pudiendo éstas ayudar en gran medida a la mejora académica y conductual de ellos, enfatizando la relación alumno- maestro, impulsando la reciprocidad en el accionar diario, sin dejar de lado la transmisión de contenidos pedagógicos, sino además formar a los estudiantes en la adquisición de habilidades afectivas y sociales esenciales para el éxito educativo.

Al educar según estos principios es necesario el conocimiento de educación emocional, para coadyuvar al proceso de formación integral de los estudiantes, ya que el intelecto no podría funcionar de manera aislada con la participación de la inteligencia emocional, siendo este un proceso que exige la coordinación armónica de ambas. Como refiere (Goleman, et al., 2012), para alcanzar el éxito en nuestra vida ya sea ésta, social, profesional y académica, nuestros actos deberían reflejar el conocimiento de habilidades emocionales, las cuales resulten en la consecución de la felicidad del individuo por cuanto al conocer, identificar y manejar proactivamente las propias emociones, estaremos en condiciones de generar relaciones inter e intrapersonales óptimas.

En tal sentido, la educación deberá garantizar el desarrollo integral de los jóvenes, asegurando así su absoluta realización autónoma y colectiva, orientado al Buen Vivir, entonces, la implicación de educación emocional en el aula a través de estrategias, va ligada o de forma subyacente hacia la consecución de aquello, fortaleciendo la vida académica y conductual de los estudiantes, considerando a éste como un eje transversal que permita desarrollar las potencialidades y capacidades de los mismos.

Al educar según estos principios es necesario el conocimiento de educación emocional, para coadyuvar a la mejora conductual y académica de los estudiantes, por cuanto el intelecto no podría funcionar de manera aislada con la participación de la inteligencia emocional, siendo este un proceso que exige la coordinación armónica de ambas. Se considera a la educación emocional, como un complemento necesario para augurar éxito en el proceso de aprendizaje en ellos, en efecto, el conocimiento y práctica de Educación Emocional, significaría que por sí mismos, descubran y regulen sus emociones de 


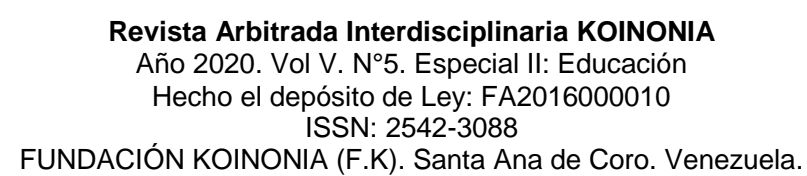

Jennifer Cecilia Ordóñez-León; Darwin Gabriel García-Herrera; Sergio Constantino Ochoa-Encalada; Juan Carlos Erazo-Álvarez

manera positiva, proyectando su práctica diaria, para que puedan ser consideradas como competencias en su vida.

Por lo tanto, el objetivo de la investigación es analizar la educación emocional y su influencia como estrategia educativa con estudiantes de bachillerato del Ecuador.

\section{Referencial teórico}

Es imperante que, en el proceso de aprendizaje de los dicentes y la consecución de habilidades, se destaque la educación emocional, la cual según estudios realizados ha producido grandes beneficios, hoy en día el reto docente no solo debería ir enfocado hacia la obtención de promedios excelentes, si no también hacia la diversificación de herramientas o estrategias metodológicas que cultiven y desarrollen la convivencia armónica entre todos los involucrados en el proceso educativo.

\section{Educación Emocional}

El termino Educación Emocional, durante mucho tiempo mantuvo un bajo perfil, sin embargo (Goleman, et al., 2012), quienes abordan dicha concepción desde la Inteligencia Emocional, convirtiéndolo en un término utilizado con frecuencia, generando interés en el contexto que se utilizare, motivando a quien lo hiciera al conocimiento de sus emociones y el manejo adecuado de las mismas para desarrollas habilidades que permitieran una convivencia armónica consigo mismo y los demás.

En concordancia con lo mencionado (Bisquerra-Alzina, 2003), aborda a la inteligencia emocional como una innovación educativa, argumentando que insta al perfeccionamiento de competencias emocionales, las cuales propenden al bienestar personal, sugiriendo que necesariamente se deben emplear y ejecutar programas que permitan llevarlas a cabo en el aula, cuyo efecto será aprender de manera motivante, ya que el conocimiento que no emociona, difícilmente se aprende.

Al considerar expuesto por (Bisquerra Alzina \& Hernández Paniello, 2017), existe esta necesidad de cultivar en los estudiantes la adquisición de su bienestar, a través de conductas que puedan generar un desarrollo óptimo no solo en su vida académica sino 


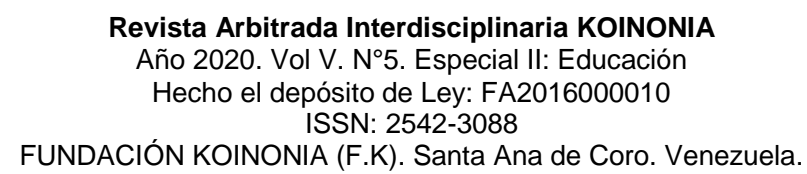

Jennifer Cecilia Ordóñez-León; Darwin Gabriel García-Herrera; Sergio Constantino Ochoa-Encalada; Juan Carlos Erazo-Álvarez

también en el ámbito social y familiar, siendo la educación emocional este gran empujón que se necesita para que los estudiantes desarrollen sus competencias al máximo, proyectando su educación hacia la consecución de un objetivo claro en la vida de cada uno de ellos, alejándolos de ciertos conflictos y/o distracciones que pudieran entorpecer dicho cometido.

\section{Motivación y aprendizaje.}

Si consideramos a la emoción como ese conector importante que infiere en la manera de como los estudiantes van aprehender sin duda, podríamos evocar un elemento de importancia vinculado a ello y es la motivación, aquella que podríamos abordar como ese impulso o el accionar, proveniente de todo aquello que emana de manera intrínseca de los educandos -sus emociones- como lo manifiesta (Boro, 2000), entonces la motivación tiene estrecha relación con ellas, de cierta manera el educando se prepara para su accionar adecuado tanto de manera física y cognitiva, enfocándonos en ésta última como aquella que regule su conducta, acercándose a situaciones que generen satisfacción y evitar aquellas que le supondrían malestar. Por ende, este acercamiento a las emociones de los estudiantes, a través de la motivación diaria, favorecerá el aprendizaje.

\section{A continuación, se presentan estudios realizados en países y la connotación que sugiere practicar Inteligencia Emocional dentro de las aulas con estudiantes de bachillerato:}

En México (Cano-Valverde \& Reyes-Ruiz, 2015), fundamentan su investigación y la importancia de la educación emocional practicada con estudiantes de Educación Media Superior, en donde concluyen que necesariamente los procesos formativos de los estudiantes deben estar acompañados por un vasto conocimiento y óptimo desarrollo de la Inteligencia emocional, los autores hacen principal énfasis en generar ambientes pacíficos, los cuales motiven a la toma de conciencia sobre las emociones propias, considerando ésta como una determinante para transformarlas, esta habilidad es 


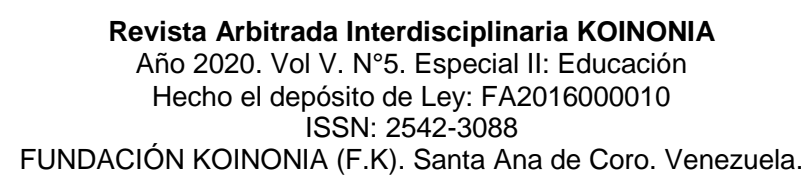

Jennifer Cecilia Ordóñez-León; Darwin Gabriel García-Herrera; Sergio Constantino Ochoa-Encalada; Juan Carlos Erazo-Álvarez

esencial impulsar en los estudiantes, para así lograr una convivencia armónica entre los implicados en el proceso educativo.

En concordancia con lo mencionado, (Segura-Martín, et al., 2015), en su investigación realizada a estudiantes de Bachillerato en un colegio de Venezuela, coinciden que existe correlación entre el desarrollo de habilidades emocionales, las cuales ejercen acción positiva en el autocontrol de conductas, esto significa que mejoraron notablemente su bienestar personal y social, además de su rendimiento académico y conductual, es así cómo, el control de sus emociones positivas y la canalización de aquellas no tan buenas, ayudaron significativamente al proceso de aprendizaje, abriendo paso para la adquisición de nuevos contenidos e información.

En la investigación realizada por (Broc-Cavero, 2019), posterior a su estudio y resultados obtenidos mediante la aplicación de test a estudiantes de educación secundaria en España, sugiere que la E.E. en cierto sentido, se le ha otorgado un valor y dimensión superior al que reflejan dichos resultados, en base a ciertas afirmaciones que correspondiendo al auge del tema mencionado, sin que hubiere una base científica que asegure su autenticidad, sin embargo, aduce que la educación emocional debe considerarse como un objetivo entre los educadores mediante el cual se denota corresponsabilidad en relación a contenidos y o aspectos emocionales a ser impartidos. Cabe señalar que, en las investigaciones antes mencionadas los autores utilizaron estrategias como relatos escritos, notas y registro de seguimiento, cuestionarios, entrevistas los cuales permitieron monitorear e inferir los resultados finales.

Es fácil comprender por qué la educación emocional ejerce un rol importante dentro del contexto educativo, cuya propuesta es optimizar el desarrollo integral del educando, instaurando una mejora en el ejercicio de enseñar, modificando incluso concepciones tradicionales, tan arraigadas en los docentes. El estudio de la educación emocional, por mucho tiempo se ha visto rezagado en Ecuador, considerándola a ésta menos importante, primando el esfuerzo docente únicamente en los contenidos académicos dejando de lado aquellos que tienen que ver con el ámbito emocional. 


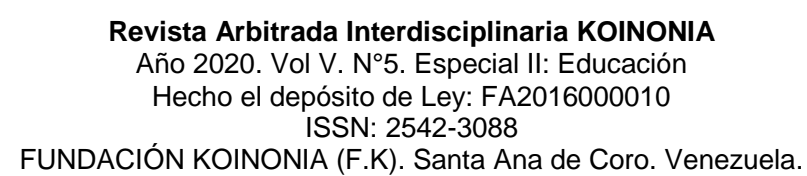

Jennifer Cecilia Ordóñez-León; Darwin Gabriel García-Herrera; Sergio Constantino Ochoa-Encalada; Juan Carlos Erazo-Álvarez

En base a esto tras la investigación efectuada por (Bravo-Vélez, et al., 2017), proponen su investigación enfocada en tres etapas y su caracterización; entre sus propuestas se denotan los ejes transversales que cada asignatura trabaja en forma sistemática, la necesidad de trabajar de manera concatenada los valores intrínsecos, aquello que se puede ser observado en el aula, de cierta manera jerarquizan la educación emocional en los estudiantes, contenidos mediante los cuales se ha de fortalecer el autoestima de los dicentes, evitando así conductas disruptivas; si bien es cierto generalmente las Instituciones Educativas están provistas de DECEs (Departamento de Consejería Estudiantil), cuyas actividades están enfocadas a cumplir ejes de acción entre ellas el acompañamiento, es menester que el docente contribuya a la formación integral de los educandos, no solo limitando su esfuerzo en el enfoque intelectual, sino necesariamente psico-emocional.

De tal manera, la educación debe orientarse hacia la consecución de una meta clara, que sería, coaccionar a sus educadores hacia la intervención, en contenidos cognitivos y emocionales, como lo expresa (Lara-Lara, 2019), en cuanto a la realidad que debe afrontar un docente desde la perspectiva inclusiva enfocado en la inclusión, como la capacitación continua en saberes no solo de índole académico, en su capacidad reflexiva a través de la investigación y perspicacia en lo que refiere a las necesidades de sus educandos, he considerado relevante su aporte, ya que motiva la labor docente hacia el interés de las emociones, deseos, sentimientos de ellos, fomentando así el aprendizaje significativo.

En un estudio realizado por (Collado-Ruano, et al., 2018), según su perspectiva hacia el desarrollo de las clases impartidas en el aula y el rol docente, llegan a la conclusión de que los maestros necesariamente requieren la aprehensión de todas las habilidades intrínsecas, que le permitan sensibilizar y agudizar su conocimiento de forma global a sus estudiantes y en la convivencia diaria denotar en ellos, la práctica de valores, participación y diálogo, respetando al otro - compañero- considerándolo como un ser íntegro y diferente a él. 


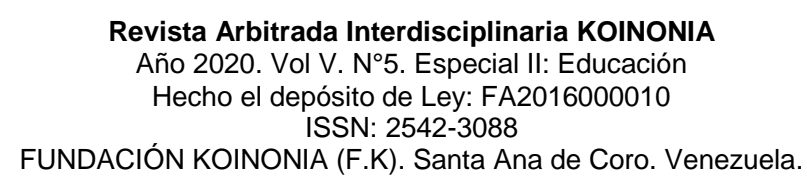

Jennifer Cecilia Ordóñez-León; Darwin Gabriel García-Herrera; Sergio Constantino Ochoa-Encalada; Juan Carlos Erazo-Álvarez

En cierto sentido, el estudio de varias disciplinas en el sistema educativo, establece que la metodología para aplicar con los estudiantes, se supone comience en el desarrollo de competencias que denoten dimensiones de la educación hacia su desarrollo integral, de tal manera que se considere la transición de los educandos en todas sus etapas y por consiguiente el respeto, tolerancia y guía en el paso de las mismas.

\section{METODOLOGÍA}

La investigación llevada a cabo fue de tipo descriptiva no experimental, su enfoque fue cuantitativo y de cohorte transversal, para su estudio se tomó la totalidad de la población o universo que estuvo conformada por 55 estudiantes del Segundo de Bachillerato de la Unidad Educactiva del Milenio "Manuela Garaicoa de Calderón”, sección vespertina, el instumento se aplicó a 51 estudiantes de la Insticuión referida anteriormente.

Esto permitió el abordaje del conocimiento de educación emocional y sus incidencias, a traves del empleo de Trait Meta Mood Scale (TMMS-24), el cual evalúa diversas dimensiones, entre ellas intrapersonales como atención, claridad y reparación emocional, como cita (González, et al., 2020), en sus investigaciones, se adicionaron 5 preguntas, dando como resultado una encuesta de 29 interrogantes.

La recolección de datos se realizó en línea a través de la plataforma Microsoft Forms en cuestionario de escala Likert, siendo validado por el coeficiente de Alfa de Chrombatch, con resultado de 0,86. La información recopilada fue analizada estadisticamente desde la estadistica descriptiva y chi cuadrado de Pearson en apoyo del programa IBM SPSS Statics versión 19 del cuál se obtuvo las tablas presentadas en los resultados.

\section{RESULTADOS}

El análisis de la normalidad de las variables que se realizó con un intervalo de confianza del $95 \%$, arrojaron como resultados que todas las variables son paramétricas .De las variables aplicadas se consideraron las preguntadas detalladas a continucación en cada tabla con su correlación existente entre ellas utilizando Chi cuadrado. 
Jennifer Cecilia Ordóñez-León; Darwin Gabriel García-Herrera; Sergio Constantino Ochoa-Encalada; Juan Carlos Erazo-Álvarez

La relación de variables sometidas a Chi cuadrado indican una hipótesis afirmativa con un valor de P 0,040, menor a 0,05, entre si los estudiantes piensan que merece la pena prestar atención a sus emociones y estado de ánimo y la importancia de la relación con sus docentes, en base a esto se puede interpretar que existe una relación entre las variables ya que los estudiantes están totalmente de acuerdo en que es necesario prestar atención a sus sentimientos y estados de ánimo, lo cual deriva de igual manera en la percepción que consideran importante la relación que mantengan con sus docentes. Ver tabla 1.

Tabla 1.

Relación entre variables Considera importante la relación con sus docentes y Pienso que merece la pena prestar atención a mis emociones y estado de ánimo.

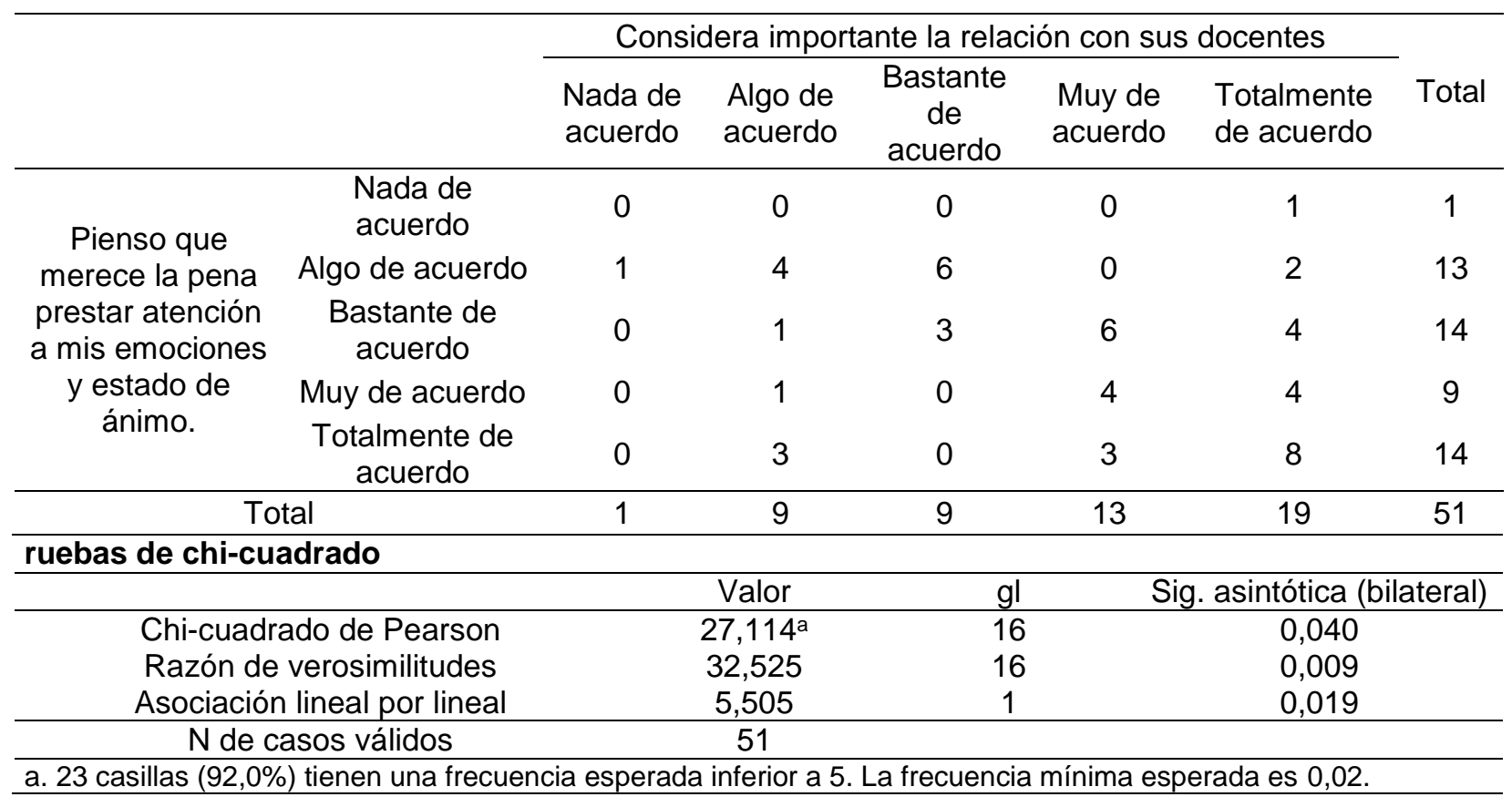

Fuente: Elaboración propia. 
Revista Arbitrada Interdisciplinaria KOINONIA

Año 2020. Vol V. N5. Especial II: Educación

Hecho el depósito de Ley: FA2016000010

ISSN: 2542-3088

FUNDACIÓN KOINONIA (F.K). Santa Ana de Coro. Venezuela.

Jennifer Cecilia Ordóñez-León; Darwin Gabriel García-Herrera; Sergio Constantino Ochoa-Encalada; Juan Carlos Erazo-Álvarez

\section{Tabla 2 .}

Relación entre Cree que su rendimiento académico y conducta mejoraría, si su docente fuera más afectivo en el aula y Tengo mucha energía cuando me siento feliz.

\begin{tabular}{|c|c|c|c|c|c|c|c|}
\hline & & \multicolumn{5}{|c|}{ Tengo mucha energía cuando me siento feliz. } & \multirow[b]{2}{*}{ Total } \\
\hline & & $\begin{array}{l}\text { Nada de } \\
\text { acuerdo }\end{array}$ & $\begin{array}{l}\text { Algo de } \\
\text { acuerdo }\end{array}$ & $\begin{array}{c}\text { Bastante } \\
\text { de acuerdo }\end{array}$ & $\begin{array}{l}\text { Muy de } \\
\text { acuerdo }\end{array}$ & $\begin{array}{l}\text { Totalmente } \\
\text { de acuerdo }\end{array}$ & \\
\hline \multirow{5}{*}{$\begin{array}{c}\text { Cree que su } \\
\text { rendimiento } \\
\text { académico y } \\
\text { conducta mejoraría, } \\
\text { si su docente fuera } \\
\text { más afectivo en el } \\
\text { aula. }\end{array}$} & Nada de acuerdo & 0 & 0 & 0 & 1 & 2 & 3 \\
\hline & \multirow{4}{*}{$\begin{array}{l}\text { Algo de acuerdo } \\
\text { Bastante de } \\
\text { acuerdo } \\
\text { Muy de acuerdo } \\
\text { Totalmente de } \\
\text { acuerdo }\end{array}$} & 0 & 6 & 0 & 1 & 3 & 10 \\
\hline & & 0 & 0 & 3 & 2 & 5 & 10 \\
\hline & & 2 & 2 & 0 & 2 & 7 & 13 \\
\hline & & 1 & 1 & 5 & 3 & 5 & 15 \\
\hline \multicolumn{2}{|c|}{ Total } & 3 & 9 & 8 & 9 & 22 & 51 \\
\hline
\end{tabular}

Pruebas de chi-cuadrado

\begin{tabular}{cccc}
\hline & Valor & gl & Sig. asintótica (bilateral) \\
\hline Chi-cuadrado de Pearson & $27,688^{\mathrm{a}}$ & 16 & 0,034 \\
Razón de verosimilitudes & 30,117 & 16 & 0,017 \\
Asociación lineal por lineal & 0,009 & 1 & 0,926 \\
\hline $\mathrm{N}$ de casos válidos & 51 & & \\
\hline a. 23 casillas (92,0\%) tienen una frecuencia esperada inferior a 5. La frecuencia mínima esperada es 0,18.
\end{tabular}

Fuente: Elaboración propia.

La Tabla 2 muestra el análisis de Chi cuadrado, donde su valor es de 0,034 menor a 0,05 , con lo cual se asume la hipótesis afirmativa considerando la relación entre percepción de los estudiantes en la creencia de que su rendimiento académico y conducta mejoraría, si su docente fuera más afectivo en el aula y su predisposición en la proyección de su energía cuando se siente feliz, dando como resultado que es imperante la disposición afectiva de los docentes para el accionar, motivación y esmero de los estudiantes en el aula de clase. 
Revista Arbitrada Interdisciplinaria KOINONIA

Año 2020. Vol V. N5. Especial II: Educación

Hecho el depósito de Ley: FA2016000010

ISSN: 2542-3088

FUNDACIÓN KOINONIA (F.K). Santa Ana de Coro. Venezuela.

Jennifer Cecilia Ordóñez-León; Darwin Gabriel García-Herrera; Sergio Constantino Ochoa-Encalada; Juan Carlos Erazo-Álvarez

\section{Tabla 3}

Relación entre Tabla de contingencia Sus maestros abordan temas de educación emocional en clases y A menudo me doy cuenta de mis sentimientos en diferentes situaciones.

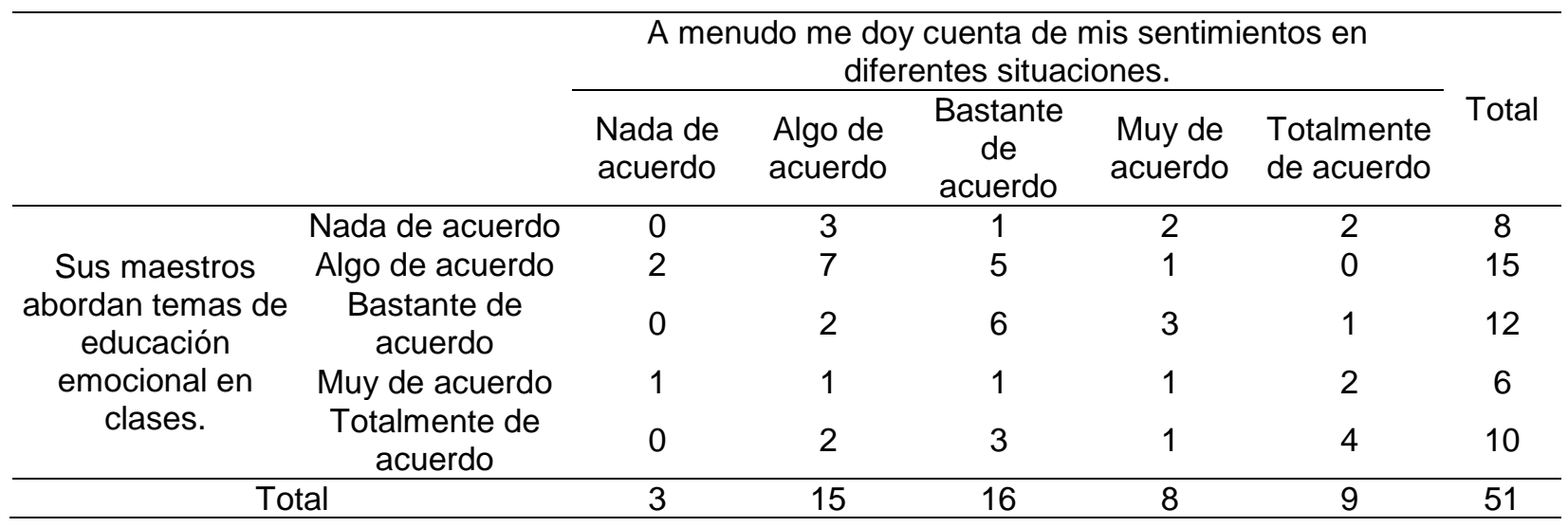

\section{Pruebas de chi-cuadrado}

\begin{tabular}{cccc}
\hline & Valor & gl & Sig. asintótica (bilateral) \\
\hline Chi-cuadrado de Pearson & $19,276^{a}$ & 16 & 0,255 \\
Razón de verosimilitudes & 22,305 & 16 & 0,134 \\
Asociación lineal por lineal & 3,218 & 1 & 0,073 \\
\hline N de casos válidos & 51 & & \\
\hline a. 25 casillas (100,0\%) tienen una frecuencia esperada inferior a 5. La frecuencia mínima esperada es $0,35$.
\end{tabular}

Fuente: Elaboración propia.

Tabla 3 muestra el análisis de Chi cuadrado, donde su valor es 0.255 mayor a 0.05 , con lo cual se asume la hipótesis nula, demostrando la no relación entre, si los estudiantes han notado que sus maestros abordan temas de educación emocional en clases y con su percepción hacia sus sentimientos en diferentes situaciones, datos significantes que demuestran la falta que los docentes aborden temas de educación emocional, para que así sus estudiantes puedan identificar sus sentimientos y al recocerlos poderlos etiquetar para accionar de manera correcta ante situaciones diversas que se presenten en la vida cotidiana. 


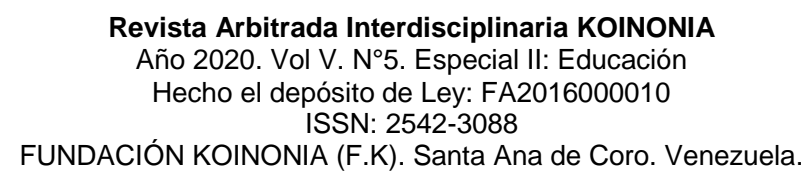

Jennifer Cecilia Ordóñez-León; Darwin Gabriel García-Herrera; Sergio Constantino Ochoa-Encalada; Juan Carlos Erazo-Álvarez

\section{PROPUESTA}

Este trabajo de investigación a través de los resultados obtenidos propone una visión específica hacia la educación emocional en los estudiantes de bachillerato considerando en primera instancia aquellos datos relevantes que han fortalecido la concepción de que es necesario un cambio de paradigma en el aula mediante la acción docente en la misma. En la puesta en pràctica de E.E.M. se realizará de la siguiente manera.

Docentes: En primer lugar, al hablar de motivación en el aula podremos manifestar que el acercamiento del docente no solo hacia el diagnóstico de conocimientos pedagógicos sino, hacia las emociones de sus estudiantes, es necesario, entonces ¿Por qué no empezar por la toma de una encuesta básica sobre ellas? Esto ayudará a generar conexión afectiva con ellos, ya que a través de sus respuestas podemos conocer cuál es el nivel de emociones o sentimientos que prevalecen en este grupo específico. Claro, el abordaje de educación emocional como estrategia utilizada por los docentes debe enfocarse en la promoción de la salud mental de ellos, no como un proceso terapéutico, sino como un propósito de mejora conductual y académica, proyectado hacia el éxito del proceso de enseñanza - aprendizaje, generando esta educación de manera transversal ya que ésta, se encuentra presente en todas las asignaturas, gestionando herramientas. ¿Cómo hacerlo? Creando un espacio propio para ello, en las planificaciones pedagógicas, pudiendo realizarlo en el tiempo de anticipación mediante ejercicios reflexivos, a través de sus anécdotas compartidas en el trato horizontal con sus estudiantes, obviamente con la predisposición y delicada capacitación de los docentes. 


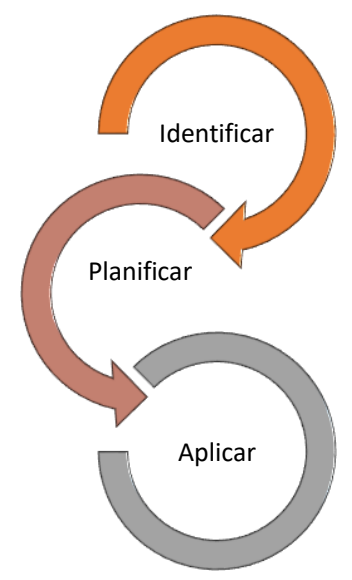

Figura 1. Proceso para implementar educación emocional en el aula.

Fuente: Elaboración propia.

Identificar. - Mediante una encuesta aplicada inicialmente los docentes podrán conocer el estado emocional de sus estudiantes y esto servirá como referente para el trabajo en el aula de aquellas emociones que requieren ser abordadas, así se creará el rapport necesario para establecer un ambiente favorable, logrando que los estudiantes perciban esa preocupación e interés por parte del maestro acerca de conocerlos más allá del ámbito pedagógico.

Planificar. - En base a los resultados obtenidos con la encuesta, se elaborará la clasificación de contenidos, se iniciará a manera de prerrequisitos con una frase motivacional que llame la atención de los estudiantes, posteriormente educar en base a la gestión de sus emociones con ejercicios reflexivos y material de apoyo, finalmente con la elaboración de un Proyecto de Vida creado por cada estudiante, para que pueda identificar aquellas fortalezas que serán de gran utilidad para la consecución de una meta final, la misma que le dotará de equilibrio emocional para alcanzarlo.

Aplicar. - Mediante un juego de roles podremos poner en práctica lo aprehendido a manera de identificar aquellas emociones que no son controladas con frecuencia y ejecutar las estrategias conocidas para lograr manejarlas.

Recomendaciones: 


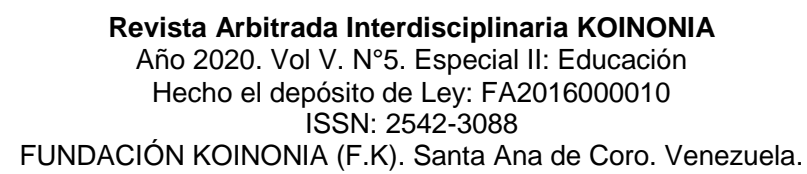

Jennifer Cecilia Ordóñez-León; Darwin Gabriel García-Herrera; Sergio Constantino Ochoa-Encalada; Juan Carlos Erazo-Álvarez

1. Si bien es cierto en bachillerato no existe una hora determinada para socializar contenido de educación emocional, podríamos dedicar una hora de clase en cada parcial para fortalecer el vínculo con nuestros estudiantes y las relaciones intra e interpersonales con sus pares.

2. Reforzar la conducta y rendimiento académico, mediante palabras de motivación constante y observación de nuestros estudiantes al momento de iniciar clases.

3. Elaborar un pequeño ensayo con los estudiantes sobre la importancia de gestionar emociones y un cuadro comparativo sobre cómo son sus sentimientos y conocimientos luego del plan ejecutado.

Estudiantes. - Es necesario propender a mejorar la calidad de vida de los estudiantes, a través de la educación emocional como una estrategia educativa, generando recursos para ellos mediante un proyecto de vida, ¿De qué manera? Pues sí, aquel estudiante que pueda determinar su proyecto de vida, ya que al hacerlo podrá enfocar sus actividades y habilidades hacia la consecución de ello, ya que cuando enfoca sus acciones, las vuelve hábitos y estarán orientados a una meta final. ¿Cómo lograrlo? En primer lugar, motivar al estudiante hacia el conocimiento de sus emociones propias, pues al hacerlo las pueden etiquetar y adelantar su forma de accionar ante determinadas circunstancias, en síntesis, descubrirse a sí mismo, mediante el conocimiento de sus habilidades e inteligencias, a través del autoconocimiento, etiquetar la emoción que siente, para que pueda regularla, al hacerlo el alumno se vuelve más empático, comprendiendo desde su unicidad para ser tolerante hacia la diversidad, generando así habilidades emocionales. 
Jennifer Cecilia Ordóñez-León; Darwin Gabriel García-Herrera; Sergio Constantino Ochoa-Encalada; Juan Carlos Erazo-Álvarez

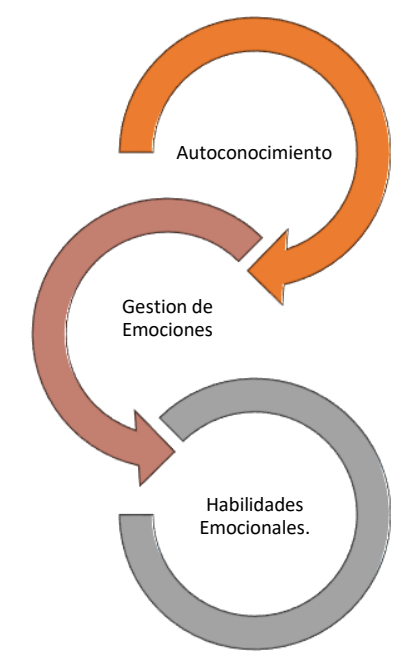

Figura 2. Proceso para implementar educación emocional en el aula. Fuente: Elaboración propia.

Autoconocimiento. - Conocer las fortalezas, cualidades y virtudes que poseen, aquello para lo que tiene mejor capacidad de lograr, sin que esto signifique avergonzarse por las limitaciones o debilidades que posea, el conocerse a sí mismo permitirá proyectar de mejor manera la reacción y relación que tenga ante sus propias emociones y ante la de los demás.

Gestión de emociones. - El conocimiento de sí mismo, permitirá identificar cuáles son sus emociones ante ciertos estímulos, si un sentimiento traerá consigo una buena conducta y una reacción aceptable y feliz, puede tener la capacidad de reproducirla constantemente. Pero, si al identificar esa emoción, es negativa y provocará ciertos conflictos internos y /o externos, es necesario etiquetarla en su mente, debido a que, al identificar ya sea ira, frustración, enojo, etc. Podrá permitirse meditar si actuar de esa manera le producirá beneficios 0 , todo lo contrario, mediante este ejercicio podrá optar por evitar darle la importancia inicial y llevarlo a la reflexión.

Habilidades emocionales. - Puesto que ahora, ha logrado conocerse a sí mismo, identificar y etiquetar sus emociones, traerán consigo la habilidad de reproducir o evitar ciertos sentimientos, que no ayuden a su equilibrio emocional, el trabajar constantemente 


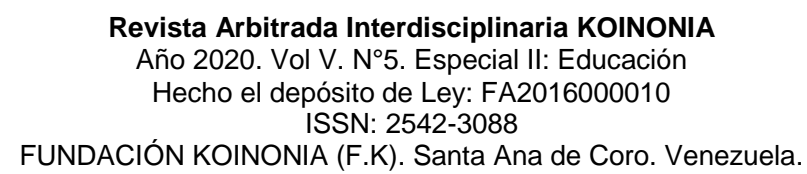

Jennifer Cecilia Ordóñez-León; Darwin Gabriel García-Herrera; Sergio Constantino Ochoa-Encalada; Juan Carlos Erazo-Álvarez

con su pensamiento asertivo lo conducirá a generar la habilidad de accionar de forma positiva para su beneficio y la de los demás, elevando su potencial individual, familiar y social, mejorando así su calidad de vida.

Recomendaciones:

1. Participar activamente de cursos que impliquen el conocimiento de fortalezas y debilidades en adolescentes.

2. No sentirse juzgado, por tener momentos de frustración, ira, enojo, tristeza, ya que estos sentimientos son tan importantes como la felicidad, sin embargo, es importante que lo reconozcan como tal, mejorando su forma de percibirlos y accionar.

3. Reconociendo que la habilidad emocional que alcance, será en base a la importancia y constancia de la gestión de sus emociones.

\section{CONCLUSIONES}

El trabajo se realizó en estudiantes del Segundo de Bachillerato de la U.E. del Milenio "Manuela Garaicoa de Calderón", presenta como resultados que la educación emocional, motivación, acercamiento afectivo y conocimiento de emociones, es considerada importante para ellos, ya que al hacerlo existe mayor predisposición hacia la percepción de contenidos, lo que llevará a la mejora académica, por ende este trato horizontal fortalecerá vínculos de afectividad entre docentes y estudiantes, generando empatía en el ámbito de enseñanza, limitando la posibilidad de romper dicho vínculo con malas actitudes, sino más bien, fortaleciendo la empatía, respeto y cordialidad entre los actores de la comunidad educativa.

La propuesta planteada de planificar E.M.M. en el aula como un eje transversal en las asignaturas impartidas en el nivel de estudio es, considerarlo como un componen te esencial que coadyuvará a la mejora académica y conductual de estudiantes, pretendiendo que su aplicación genere replicas satisfactorias en aquellos docentes que aun $\mathrm{n}$ o han tenido el acercamiento hacia dicho conocimiento. 
Jennifer Cecilia Ordóñez-León; Darwin Gabriel García-Herrera; Sergio Constantino Ochoa-Encalada; Juan Carlos Erazo-Álvarez

\section{FINANCIAMIENTO}

No monetario

\section{AGRADECIMIENTO}

A la Unidad Educactiva del Milenio "Manuela Garaicoa de Calderón"; por apoyar el desarrollo de la investigación.

\section{REFERENCIAS CONSULTADAS}

Ariza-Hernández, M. L. (2017). Influencia de la inteligencia emocional y los afectos en la relación maestro-alumno, en el rendimiento académico de estudiantes de educación superior [The Influence of Emotional Intelligence and Affection]. Educación y Educadores, 20(2), 193-210. doi:10.5294/edu.2017.20.2.2

Barrientos-Fernández, A., Sánchez-Cabrero, R., \& Arigita-García, A. (2019). Formación emocional del profesorado y gestión del clima de su aula [Teachers' emotional training and management]. Praxis \& Saber, 10(24), 119-141. http://dx.doi.org/10.19053/22160159.v10.n25.2019.9894.

Bisquerra-Alzina, R. (2003). Educación emocional y competencias básicas para la vida [Emotional Education and Basics Skills for life]. Revista De Investigación Educativa, 21(1), 7-43.

Bisquerra-Alzina, R., \& Hernández-Paniello, S. (2017). Positive psychology, emotional education and the happy classrooms program [Psicología positiva, educación emocional y el Programa Aulas Felices]. Papeles del Psicólogo, 38(1), 58 - 65.

Boro, J. C. (2000). The neuropsychology of emotion[La Neurosicología de la Emoción]. Oxford: University Press. Recuperado de https://n9.cl/w247

Bravo-Vélez, L. E., Amayuela-Mora, G., \& Colunga-Santos, S. (2017). Tendencias históricas del proceso de educación emocional en los estudiantes del bachillerato en Ecuador [Historical trends of process of emotional education process of high school students in Ecuador]. Mendive. Revista de Educación, 15(3), 305-315. 
Revista Arbitrada Interdisciplinaria KOINONIA

Año 2020. Vol V. N5. Especial II: Educación

Hecho el depósito de Ley: FA2016000010

ISSN: 2542-3088

FUNDACIÓN KOINONIA (F.K). Santa Ana de Coro. Venezuela.

Jennifer Cecilia Ordóñez-León; Darwin Gabriel García-Herrera; Sergio Constantino Ochoa-Encalada; Juan Carlos Erazo-Álvarez

Broc-Cavero, M. A. (2019). Inteligencia Emocional y Rendimiento Académico en alumnos de Educación Secundaria Obligatoria [Emotional Inteligence and Academic Permormance in Students of Compulsory SecondaryEducation]. Revista Española De Orientación y $\quad$ Psicopedagogia., $\quad 30(1), \quad 75-92$. https://doi.org/10.5944/reop.vol.30.num.1.2019.25195

Cano-Valverde, M., \& Reyes-Ruiz, M. T. (2015). Educación Emocional para vivir en paz, Estudiantes de Educación Superior [Emotional To Live In Peace Education Middle School Students]. Ra Ximhai, 11(1), 209-222.

Collado-Ruano, J., Madroñero Morillo, M., \& Álvarez González, F. J. (2018). Educación transdisciplinar: formando en competencias para el buen vivir [Transdisciplinary education: training in skills for the good living]. Ensaio: Avaliação e Políticas Públicas em Educação, 26(100), 619-644. https://doi.org/10.1590/s0104$\underline{40362018002601487}$

Goleman, D., Bennett, L., \& Barlow, Z. (2012). How Educators Are Cultivating Emotional, Social, and Ecological Intelligence [Cómo los educadores están cultivando la Inteligencia Emocional, Social y Eecológíca]. John Wiley \& Sons, Incorporated.

Gonzalez, R., Custodio, J. B., \& Abal, F. J. P. (2020). Propiedades psicométricas del Trait Meta-Mood Scale-24 en estudiantes universitarios argentinos: Propiedades psicométricas del Trait Meta-Mood Scale-24 en estudiantes universitarios argentinos [Psychometric properties of the Trait Meta-Mood Scale-24 in Argentine university students: Psychometric properties of the Trait Meta-Mood Scale-24 in Argentine university students]. Psicogente, 23(44), 1-26. https://doi.org/10.17081/psico.23.44.3469

Lara-Lara, F. (2019). Sumak Kawsay y Educación Inclusiva en Ecuador: una propuesta desde el personalismo [Sumak Kawsay and Inclusive Education in Ecuador: a proposal from personalism]. Acta Scientiarum. Education, 41(1), e40274. https://doi.org/10.4025/actascieduc.v41i1.40274

Organización de las Naciones Unidas para la Educación, la Ciencia y la Cultura [UNESCO]. (2020). Situación Educativa de América Latina y el Caribe: Hacia la educación de calidad para todos al 2015 [Educational Situation of Latin America and the Caribbean: Towards quality education for all by 2015]. Recuperado de https://n9.cl/22t6 
Revista Arbitrada Interdisciplinaria KOINONIA

Año 2020. Vol V. N5. Especial II: Educación

Hecho el depósito de Ley: FA2016000010

ISSN: 2542-3088

FUNDACIÓN KOINONIA (F.K). Santa Ana de Coro. Venezuela.

Jennifer Cecilia Ordóñez-León; Darwin Gabriel García-Herrera; Sergio Constantino Ochoa-Encalada; Juan Carlos Erazo-Álvarez

Segura-Martín, J. M., Cacheiro-González, M. C., \& Dominguez-Garrido , M. (2015). Estudio sobre las habilidades emocionales de estudiantes venezolanos de bachillerato y formación técnica superior [A Study of the Emotional Skills of Venezuelan]. Educación y Educadores., 18(1), 9-26.

(C2020 por los autores. Este artículo es de acceso abierto y distribuido según los términos y condiciones de la licencia Creative Commons Atribución-NoComercial-Compartirlgual 4.0 Internacional (CC BY-NC-SA 4.0) (https://creativecommons.org/licenses/by-nc-sa/4.0/). 